\title{
Actinomicosis torácica como diagnóstico diferencial de neoplasia: a propósito de un caso
}

Nicolás Pereira, Pedro Cuevas, Camila Valencia, Javier Vega, Iván Gallegos, Jaime Gonzalo Fernández y Jorge Salguero

Thoracic actinomycosis in the differential diagnosis of neoplasm:

$$
\text { a propos of a case }
$$

Actinomycosis is an infrequent infection caused by bacteria from Actinomyces genus that manifests as a chronic, suppurative and progressive disease. It's more common in men. Thoracic actinomycosis occurs in $15 \%$ of the cases, and infection of the chest wall is less frequent. The clinical presentation mimics tuberculosis or neoplastic processes. In this article we present the case of a 63 year-old man with no comorbidity, with pulmonary actinomycosis involving the chest wall mimicking a neoplastic process, basing the diagnosis on histopathologic findings.

Key words: Actinomyces spp., thoracic actinomycosis, lung neoplasm.

Palabras clave: Actinomyces spp., actinomicosis torácica, neoplasia pulmonar.

\section{Introducción}

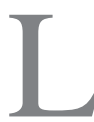

a actinomicosis es una infección crónica, supurativa y progresiva asociada a una mala higiene oral; es más frecuente en hombres (3:1) y en pacientes inmunocomprometidos ${ }^{1-4}$. Parece ser menos común en niños y adultos jóvenes debido al tratamiento oportuno de las caries y a la ausencia de enfermedad periodontal ${ }^{5}$. A pesar de la inmunosupresión celular y humoral que acompaña al SIDA, la actinomicosis es infrecuente en la población infectada por VIH.

La actinomicosis es causada por bacterias del género Actinomyces, bacilo grampositivo ramificado, anaerobio facultativo. Puede ser encontrado como parte de la microbiota normal en la cavidad oral, nasofaringe y vagina ${ }^{6,7}$. La enfermedad es caracterizada por la formación de abscesos y trayectos fistulosos junto con la descarga de material purulento que contienen gránulos amarillos sulfurosos ${ }^{6-8}$.

La actinomicosis de localización torácica se presenta en alrededor de $15 \%$ de los $\operatorname{casos}^{6-8}$ y el compromiso de la pared torácica es una manifestación aún más infrecuente correspondiendo a $12 \%$ de los casos de las actinomicosis torácicas ${ }^{5}$. La forma pulmonar es causada probablemente por la aspiración pulmonar de secreciones de la orofaringe o del tracto gastrointestinal ${ }^{1,5}$. La presentación clínica puede simular procesos neoplásicos o tuberculosis.

A continuación se presenta el caso de un paciente con actinomicosis pulmonar con compromiso de la pared torácica que simulaba un proceso neoplásico.

\section{Caso clínico}

Paciente de 63 años, de sexo masculino, con historia de tabaquismo crónico, sin otros antecedentes mórbidos.
Consultó por dolor en el hemitórax izquierdo de 3 semanas de evolución, asociado a una pérdida de peso no cuantificada y a un aumento de volumen sensible en relación al músculo pectoral izquierdo. La radiografía de tórax mostró una opacidad de bordes mal definidos en la región perihiliar izquierda (Figura 1A). Se solicitó una TAC de tórax que evidenció un tumor de 6,3 por 5,8 cm en el lóbulo pulmonar superior izquierdo (flecha larga Figura 1B) con compromiso extenso de la pared torácica anterior, sin signos de invasión ni compromiso de estructuras bronquio-vasculares centrales, sugerente de una lesión neoplásica broncogénica periférica del lóbulo superior izquierdo asociado a adenopatías hiliares y mediastínicas ipsilaterales (flecha corta Figura 1B). Con estos hallazgos, se decidió realizar una fibrobroncoscopia que mostró un leve engrosamiento de la carina, língula y lóbulo superior izquierdo, sin lesiones intraluminales. Se tomaron tres muestras transbronquiales cuya biopsia se informó como una bronquitis crónica inespecífica con metaplasia escamosa incompleta. Fue internado para completar su estudio evolucionando con dolor persistente en relación a la masa tumoral, sin fiebre y con hemodinamia estable. Dentro de los exámenes de laboratorio destacaba una anemia (hemoglobina 9,5 g/dl), trombocitosis (776.000 $/ \mathrm{mm}^{3}$ ) y leucocitosis con desviación izquierda (42.600/ $\mathrm{mm}^{3}$ con $37 \%$ de baciliformes). Se realizó un mielograma que mostró una hiperplasia mieloide compatible con una médula reactiva sin constituir una neoplasia hematológica.

Se realizó una punción percutánea por radiología intervencional que dio salida a contenido purulento, cuyo cultivo bacteriano aeróbico fue negativo. Por sospecha de tumor de la pared costal abscedado, se realizó un drenaje quirúrgico del absceso, aseo y desbridamiento del tejido desvitalizado. Se tomó una muestra de la pared torácica
Hospital Clínico Universidad de Chile.

Departamento de Cirugía (NP, PC, $J F, J S)$.

Departamento de Medicina Interna (CV).

Médico cirujano, Universidad de Chile (JV).

Departamento de Anatomía Patológica (IG).

Recibido: 7 de agosto de 2011 Aceptado: 3 de mayo de 2012

Correspondencia a: Nicolás Pereira C. nicolaspereirac@gmail.com 


\section{Caso Clínico}

Figura 1. Radiografía (A) y CT de tórax (B) al ingreso.

Figura 2. Biopsia de tejido de pared torácica. A. Tinción HE, (100x). Colonia de Actynomices (flecha) englobada por el infiltrado inflamatorio al que se agregan polimorfonucleares neutrófilos. B. Tinción PAS, (400x). Colonia de Actinomyces con tinción de color magenta, notar filamentos radiados (flecha).
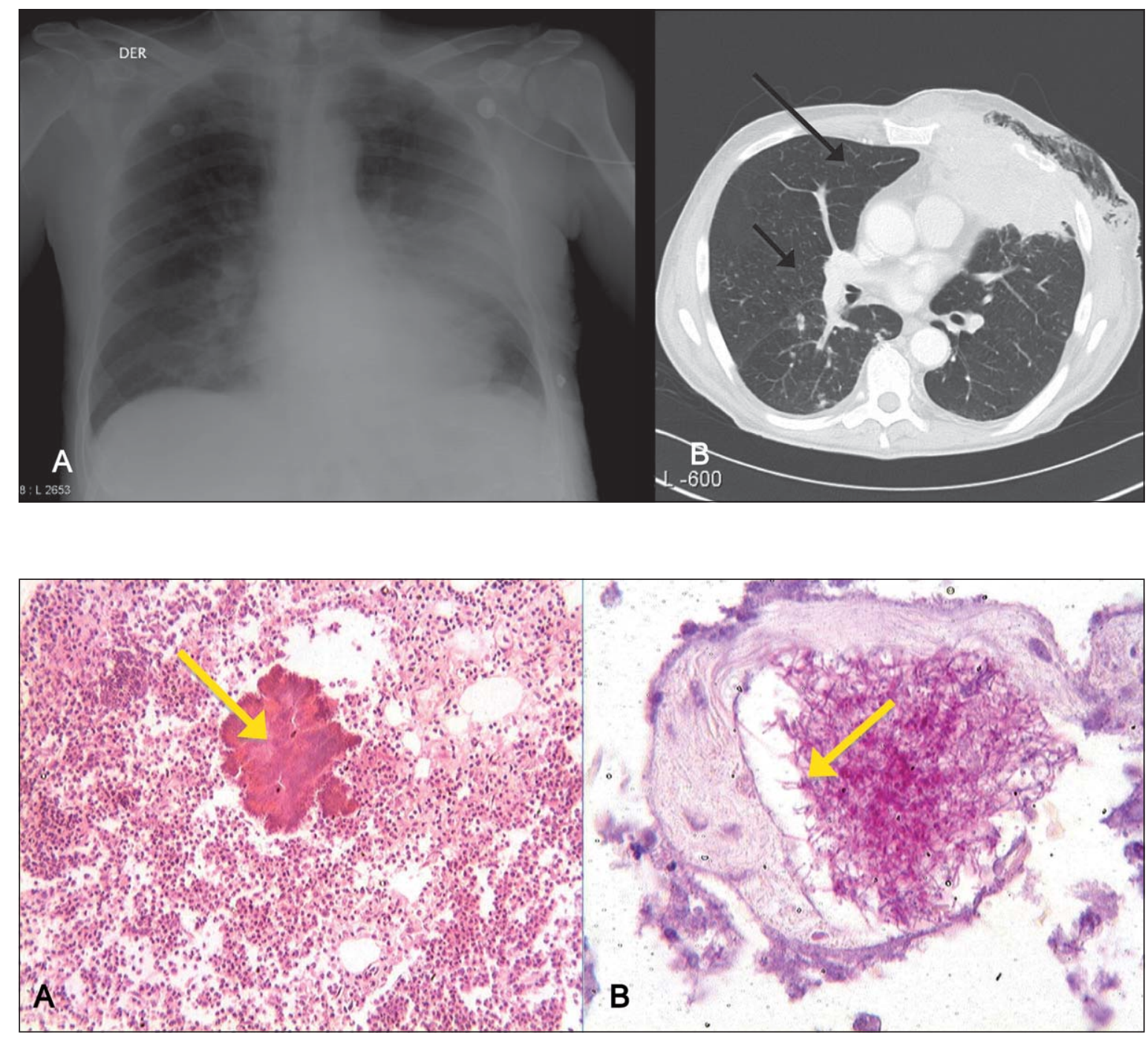

para biopsia y se instaló un sistema aspirativo para cierre de la herida.

La biopsia fue informada como una actinomicosis de la pared torácica, con compromiso de piel, tejido subcutáneo, músculo pectoral y porción perióstica de la costilla, sin elementos histológicos malignos (Figura 2). Fue evaluado por el equipo de infectología decidiéndose tratamiento antimicrobiano con penicilina $\mathrm{G}$ sódica i.v. durante 2 semanas. Evolucionó en buenas condiciones generales, con regresión de los parámetros inflamatorios y disminución del débito de los drenajes. A las tres semanas postoperatorias se realizó el último aseo quirúrgico y cierre definitivo de la herida.

Se dio de alta con amoxicilina hasta completar tres meses. Se mantuvo en controles ambulatorios, sin evidencias de complicaciones y con control radiológico a los siete meses postoperatorios que mostró regresión completa de la lesión (Figura 3).

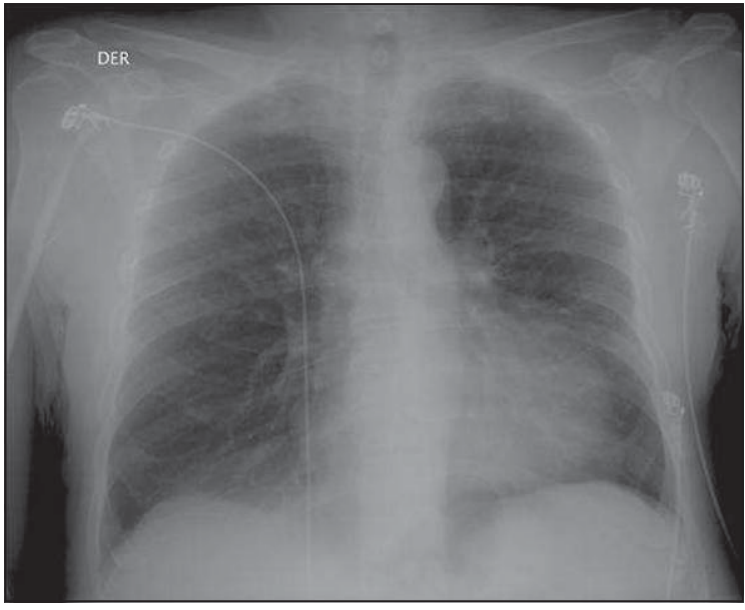

Figura 3. Radiografía de tórax de control (7 meses postoperatorio). 


\section{Discusión}

La actinomicosis es una enfermedad poco común causada por bacterias anaerobias facultativas del género Actinomyces, las cuales son comensales de la cavidad oral y gastrointestinal ${ }^{9}$. La especie más frecuente es Actinomyces israelii, aunque otras como A. meyeri también pueden causar enfermedad ${ }^{10}$. Hay escasos registros sobre la incidencia de la actinomicosis, pero al parecer ha disminuido francamente en las últimas décadas. La forma de presentación de la actinomicosis también ha cambiado. Actualmente, se presenta de manera menos agresiva comparado con la era pre-antimicrobiana. Estos cambios en la incidencia y forma de presentación pueden también ser resultado de una mejora en la higiene oral y mayor disponibilidad e inicio precoz de antimicrobianos cuando se sospecha una infección pulmonar ${ }^{10}$. En nuestro país existen sólo revisiones del tema en el área ginecológica y de cabeza y cuello ${ }^{11-13}$, por lo que nuestro caso constituye una experiencia no antes comunicada en nuestro medio.

Existen tres formas principales de actinomicosis: cérvico-facial (65\%), abdominal (20\%), y torácica (15\%). La actinomicosis torácica usualmente compromete pulmones, pleura, mediastino y pared torácica. Los hombres son afectados tres a cuatro veces más que las mujeres ${ }^{14}$. La infección torácica es consecuencia de la aspiración de microorganismos propios de la boca y orofaringe en pacientes entre la quinta y sexta década de vida, con factores predisponentes como una pobre higiene oral ${ }^{15}$. Probablemente, el mayor número de casos en este grupo etario se debe a una mayor incidencia de co-morbilidades como: diabetes mellitus, tabaquismo, alcoholismo, neoplasias, enfermedades pulmonares crónicas, inmunosupresión, deterioro en la dentadura y enfermedad periodontal ${ }^{16-18}$. De manera común, la infección se origina en el parénquima pulmonar y puede progresar a la pared torácica directamente extendiéndose a través de la pleura ${ }^{14}$. La infección de la pared torácica puede llevar a la aparición de manifestaciones cutáneas típicas con trayectos fistulosos y descarga purulenta.

La actinomicosis limitada a la pared torácica, sin evidencia de compromiso pulmonar ni de otros sitios en extremadamente rara. Se postulan varios mecanismos patogénicos: por diseminación hematógena que puede alcanzar tejidos blandos de cualquier localización incluyendo la pared torácica, la extensión desde una actinomicosis cervical o abdominal que puede comprometer la pared torácica o como resultado de una lesión pulmonar primaria resuelta espontáneamente o con tratamiento antimicrobiano. En estos casos, una masa en la pared torácica puede ser frecuentemente confundida con un tumor maligno, ya sea un carcinoma primario con compromiso de la pared, un linfoma o un sarcoma primario. La actinomicosis torácica también puede estar asociada a tumores malignos ${ }^{9,10}$. Otras infecciones como tuberculosis, aspergilosis o abscesos piógenos por Staphylococcus aureus o Klebsiella sp. pueden manifestarse como un proceso torácico aislado y deben ser incluidos como diagnóstico diferencial.

El diagnóstico se basa en los hallazgos histopatológicos, siendo necesario para el diagnóstico definitivo el aislamiento del microorganismo en cultivos desde una muestra de pus, tejido o gránulos sulfurosos ${ }^{19}$. Sin embargo, el rendimiento de los cultivos es bajo $(<50 \%)$ por varias razones: tratamiento antimicrobiano previo, sobrecrecimiento de otros microorganismos o la utilización de una metodología inadecuada ${ }^{20}$. Actinomyces spp. es un microorganismo de crecimiento lento que debe ser cultivado en un medio de agar selectivo a $37^{\circ} \mathrm{C}$ en anaerobiosis por al menos tres semanas ${ }^{19}$. Por esta razón, el médico debe comunicar al laboratorio de microbiología la sospecha de actinomicosis para que se incube por un tiempo prolongado y en el medio adecuado.

La biopsia por aspiración con aguja fina o biopsia core, puede mostrar sólo células inflamatorias sin revelar el agente infeccioso; es por esto, que la biopsia quirúrgica tiene mayor utilidad en el diagnóstico. La presencia de bacilos filamentosos grampositivos y gránulos sulfurosos en el examen histológico apoya fuertemente el diagnóstico de actinomicosis (Figura 2), como en nuestro paciente; sin embargo, estos hallazgos no son específicos de esta patología ya que se pueden encontrar en otras enfermedades, tales como nocardiosis, cromomicosis y botriomicosis ${ }^{21}$. Se necesitan tinciones especiales como Gram, GomoriGrocott (metanamina-plata) y Giemsa para demostrar el microorganismo grampositivo filamentoso ramificado en la periferia de los gránulos ${ }^{22}$. En este caso el paciente se recuperó con el drenaje y el tratamiento antimicrobiano con penicilina, lo que no habría sido suficiente para las otras etiologías con histología similar, hecho apoya claramente el diagnóstico de actinomicosis.

Varios autores recomiendan administrar penicilina G intravenosa por un mes y luego reemplazarla por penicilina oral por 6 a 12 meses o por amoxicilina ${ }^{1,4,16,17}$. En caso de alergia a la penicilina, se pueden utilizar sulfonamidas, clindamicina, eritromicina o cloranfenicol, así como tetraciclina y cotrimoxazol ${ }^{16}$.

En conclusión, una masa en la pared torácica, aún en ausencia de hallazgos pulmonares o cutáneos, puede ser una forma de presentación de la actinomicosis; una combinación de estudios histológicos, microbiológicos y moleculares apropiados pueden maximizar las probabilidades de tener éxito en el diagnóstico.

\section{Resumen}

La actinomicosis es una infección poco común causada por bacterias del género Actinomyces que se manifiesta como una enfermedad crónica, supurativa y progresiva. 
Es más frecuente en hombres. La actinomicosis torácica se presenta en $15 \%$ de los casos, siendo el compromiso de pared torácica aún menos frecuente. La presentación clínica simula procesos neoplásicos o tuberculosis. Se describe el caso de un hombre de 63 años, sin co-morbilidad, con una actinomicosis pulmonar con compromiso de pared torácica que simuló ser un proceso neoplásico. El diagnóstico fue confirmado por los hallazgos histopatológicos.

\section{Referencias bibliográficas}

1.- Bakhtawar I, Schaefer R F, Salian N. Utility of Wang needle aspiration in the diagnosis of actinomycosis. Chest 2001; 119: 1966-8.

2.- Chouabe S, Perdu D, Deslée G, Milosevic D, Marque E, Lebargy F. Endobronchial actinomycosis associated with foreign body. Chest 2002; 121: 2069-72.

3.- Coodley E L, Yoshinaka R. Pleural effusion as the major manifestation of actinomycosis. Chest 1994; 106: 1615-7

4.- Enders M, Reitnauer K, Girmann M, Lindemann W, Ukena D, Sybrecht G W. Thoracic actinomycosis - a case report. Pneumologie 1999; 53: 216-8.

5.- de la Espina MA, López-Menendez C, RuizMartínez R, Molino-Trinidad C. Pulmonary actinomycosis with thoracic soft tissue mass: a rare onset form. Eur J Radiol 2001; 37: 195-9.

6.- Tastepe A I, Ulasan N G, Liman S T, Demircan S, Uzar A. Thoracic actinomycosis. Eur J Cardiothorac Surg 1998; 14: 578-83.

7.- $\quad$ Hsieh M J, Liu H P, Chang J P, Chang C H. Thoracic actinomycosis. Chest 1993; 104: 366-70.
8.- Beier K H, Rusnak R A. Unusual presentation of cervicothoracic actinomycosis complicated by pericardial effusion: a case report. J Emerg Med 1997; 15: 303-7.

9.- Kapotsis G E, Daniil Z, Klimopoulos S, Malagari K, Roussos C, Rontogianni D, et al. A painful and swollen right breast in a young male. Eur Respir J 2004; 24: 1066- 8.

10.- Mabeza G F, Macfarlane J. Pulmonary actinomycosis. Eur Respir J 2003; 21: 545-51.

11.- Torres S, Schalper K, Piérart C. Análisis de la presencia de actinomicosis pélvica en mujeres de una comunidad rural en Chile. Rev Chil Obstet Ginecol 2002; 67: 232-6.

12.- Madrid F, Díaz S, Mucientes F, Klaassen R. Actinomicosis ginecológica. Rev Chil Obstet Ginecol 2003; 68: 21-7.

13.- Schmidt T, Caselli G, Díaz R. Actinomicosis del hueso maxilar superior. Rev Otorrinolaringol Cir Cabeza Cuello 2003; 63: 127-31.

14.- Kwong J S, Muller N L, Godwin J D, Aberle D, Grymaloski M R. Thoracic actinomycosis: CT findings in eight patients. Radiology 1992; 183: 189-92.

15.- Webb W R, Sagel S S. Actinomycosis involving the chest wall: CT findings. Am J Roentgenol
1982; 139: 1007-9.

16.- Chouabe S, Perdu D, Deslée G, Milosevic D, Marque E, Lebargy F. Endobronchial actinomycosis associated with foreign body: four cases and a review of the literature. Chest 2002; 121: 2069-72.

17.- Kobashi Y, Yoshida K, Miyashita N, Niki Y, Matsushima T. Thoracic actinomycosis with mainly pleural involvement. J Infect Chemother 2004; 10: 172-7.

18.- Endo S, Murayama F, Yamaguchi T, Yamamoto S, Otani S, Saito N, et al. Surgical considerations for pulmonary actinomycosis. Ann Thorac Surg 2002; 74: 185-90.

19.- Wong V K, Turmezei T D, Weston V C. Actinomycosis. BMJ 2011; 343:d6099.

20.- Bennhoff D F. Actinomycosis: diagnostic and therapeutic considerations and a review of 32 cases. Laryngoscope 1984; 94: 1198-217.

21.- Smego R A Jr, Foglia G. Actinomycosis. Clin Infect Dis 1998; 26: 1255-61

22.- Russo T A. Agents of actinomycosis. In: Mandell GL, Bennett JE, Dolin R, eds. Principles and Practice of Infectious Diseases 7th ed. Elsevier Churchill Livingstone, 2010: 3209-19. 